\title{
Fernand Dorais, hypnotiseur et illusionniste
}

\author{
François Paré
}

(University of Guelph)

I est sans doute l'un des plus importants penseurs de l'Ontario français. Mais, en même temps, cet homme est le symbole de notre absence de nom, de notre absence de renom. Il est notre témoin, notre scribe, notre chroniqueur. Depuis vingt-cinq ans, Fernand Dorais raconte l'histoire, toujours la sienne propre, de notre naissance à la théorie. C'est l'histoire "bizarre des enfants bannis de la ville par leurs parents," comme le dira prophétiquement Raymond Quatorze, dont le roman flamboyant vient de nous heurter de plein fouet. ${ }^{1}$ Car, pour le meilleur et pour le pire, Fernand Dorais s'est institué lui-même l'interprète aérien de la "prison rose-bonbon." "Nos pères, nos mères ont honte de nous, ils nous ont en aversion. Ils nous haïssent, vous vous rendez compte! Nous avons juré de les tuer, de les anéantir si jamais nous recouvrons le pouvoir de voler. C'est pour ça qu'ils nous ont séquestrés ici, dans la clairière, aux portes de Barnumbourg." ${ }^{2}$ Je ne sais trop pourquol en ce moment La Prison rose bonbon évoque tant pour moi le discours théorique de Fernand Dorais; peut-être est-ce simplement la clairière et une évocation de la lumière; peut-être est-ce plutôt l'attente exaspérée aux abords de la ville fictive. Peut-être Dorais joue-t-il encore dans notre culture si théâtrale le rôle d'Yvan Lualaba, le personnage louche mis en scène par Raymond Quatorze, le rôle de l'extraordinaire duplicité: LE GRAND DORAIS, HYPNOTISEUR ET ILLUSIONNISTE. ${ }^{3}$

Je ne tracerai pas de lignes biographiques ici, sinon pour indiquer ceci: professeur à l'Université Laurentienne de Sudbury, depuis 1969, Dorais est dès son arrivee assimilé aux forces vives de la culture, dont il devient l'un des tèmoins privilégiés. «Enfant perdu de la Grande Dépression, fils du clérico-nationalisme," il n'abandonnera jamais vraiment son rôle de clerc, restant, comme s'il en était le secrétaire timide, dans les marges de la contre-culture et de la nouvelle littérature franco-ontarienne. Il prend note. Ses oeuvres ne sont que des pré-textes, de simples témoignages de celui 
qui n'a pas et n'aura pas de domicile fixe. ${ }^{4}$ Il réserve ses plus amères invectives pour l'Université Laurentienne à qui il reproche sa "mentalité de comptable agréé". ${ }^{5}$ Il participe aux premiers moments des éditions Prise de Parole, de CANO, la Coopérative des artistes du Nouvel-Ontario, des cuisines de la poésie, des Nuits sur l'Étang. Il a lui-même plusieurs fois raconté cette expérience, de sorte qu'il n'est pas utile de revenir à ces éléments biographiques.

Mais, c'est par son sens de l'observation sociale et surtout son goût de la mystification que Dorais importe pour nous. Il devient, dès ses premières interventions en ce sens en 1978 l'un des plus éloquents architectes de la toute nouvelle institution littéraire franco-ontarienne, dont le centre géographique serait, et sera pour un temps, la région sudburoise. Il s'en fait l'écho, parfois pessimiste, à plus d'un moment dans ses deux ouvrages, surtout dans Entre Montréal et Sudbury, beaucoup plus fidèle à l'expérience franco-ontarienne, et dans un article inédit, où le je du narrateur-chroniqueur s'est métamorphosé en simple ça, objectifié et objectifiant, traçant les limites de notre "horizon commun d'intelligibilité."

Ce que propose Fernand Dorais au cours des années, c'est une véritable anthropologie de la minorisation. D'autres avaient parlé, ailleurs, de colonialisme, d'oppression culturelle, de double discours, de déshumanisation; Dorais, lui, envisage la vie culturelle minoritaire, dont l'Ontario français est pour lui l'exemple stratégique, comme un ensemble de conditions radicalement spécifiques, qui déterminent et justifient les individus jusque dans leur identité et leur appartenance à une origine dans le sens. Car c'est cette origine qui est absolument problèmatique. Dorais ne cesse depuis vingt-cinq ans d'en faire la narration comme si ce récit syncopé allait ultimement en exorciser la terrible méprise. Le minoritaire, parce qu'il est à tous points de vue minorisé, n'a pas d'origine, se caractérise par ce manque même, qui le laisse alors fatalement condamné à une incessante dérive. Il est humilié, bafoué, vidé de son appartenance au sens, marginalisé, réduit au silence. Mais, voilà le miracle, ce comble de l'humiliation est son identité, le lieu désintégré de sa participation, sinon originelle, du moins originale, à la parole.

Il faut relire à ce sujet le "Document 4" d'Entre Montréal et Sudbury, un texte important où Dorais s'insère dans la tradition de 
l'écrit anti-colonialiste, celui de Fanon et de Memmi. ${ }^{7}$ Je me permets de citer un assez long extrait de ce document, ne serait-ce que pour démontrer la violence de l'argumentation, lorsqu'elle s'applique à la socio-politique de l'Ontario français, peuple de "névrosés." Qui sontils? Qui sommes-nous?

Les nèvrosés, ceux qui d'entrée de jeu ont tort de n'avoir pas réussi. Les Humiliés de la planète, l'Échec de notre histoire, la poubelle du cosmos. Qui, il le faut bien, prennent figures historiques de dëlinquants, déviants, mésadaptės, arriérés, fous; puis d'opprimés, de laissés pour compte; les minoritaires, les underground, les acculturés; bref les marginalisés, qui prenaient nom en terre américaine d'Indiens, Nègres, chicanos, Esquimaux, féministes, gais ... et Canuks, et dans cet ordre. Ceux qui, de l'intérieur se montrent tout déstructurés de langage, de pensée et d'action: les "pas d'allure" qui ne "sont pas sortables" (EMS, p.62).

Cette longue énumération, qui se présente en fait comme une procession interminable des pauvres, indique très clairement la place exemplaire de la minorisation parmi tous les cas criants de l'oppression. Cette procession se clôt naturellement par le groupe des Canuks, "les pas d'allure," les "pas sortables," alors que la violence des invectives se tourne maintenant contre le minoritaire lui-même, le déstructure, le désarticule. Il est à la fin pour Dorais incapable de produire du sens, du sens sensé tout au moins. Il n'a plus que son humiliation pour s'affirmer.

Or Dorais exprime cette dépossession en termes psychologiques. Ici, il s'agira d'une névrose, ailleurs d'une carence affective, ailleurs encore d'une peur panique de sortir, de dire la vérité. C'est que le minoritaire est une personne infantilisée et qu'au fond il n'y a que le cadre de références de la psychologie pour en parler convenablement. Le Franco-ontarien dans ce qu'il a de plus typique est un spectateur de sa propre existence, coupé d'une véritable intervention dans le monde qui l'entoure et le détermine. Le découragement quasi 
congénital a entraîné son naufrage dans l'indiffërence. "Ainsi castré, émasculé, le Franco-ontarien n'a pu connaître que le régime de la conduite fausse, que le système du mensonge affectif, que lui ont imposé les yeux de coulisse de la politique, la politicaillerie grise et ennuyeuse, gouvernementale, dont seul le parlementarisme a fondé puis refondé ce pays dans l'indifférence totale de ses citoyens" (EMS, p.90). ${ }^{8}$ Il est facile de se laisser emporter par la crue de ces images masculines de mutilation. Elles habitent et motivent tout le discours de Fernand Dorais sur l'expérience minoritaire et font de ses articles, surtout avant 1980, les textes sans doute les plus violents que le Canada français ait jamais produits sur cette question. Qu'il y ait là une certaine exagération, un certain lyrisme de la dépossession, cela ne fait aucun doute. Mais ce qui importe, c'est que les prèmisses de la minorisation, en tant que condition de mutilation, sont devenues depuis vingt-cinq ans la base de discours littéraire franco-ontarien et le lieu de ses revendications, bien au delà des textes de Dorais.

Pourtant, quoiqu'elle soit imprégnée de pessimisme, la pensée de Fernand Dorais cherche toujours l'action. Et il se trouve assez curieusement que l'espoir, très nietzschéen, surgit au terme de la plus grande déchéance. Dorais reprend donc explicitement les termes de l'absolu cyclique, trouvé dans Zarathoustra, et voit dans le minoritaire émasculé les conditions paradoxales de la libération, personnelle et collective. Car les Humiliés seront appelés à la Fête. Au delà des souffrances collectives dont ils sont les victimes, les Franco-ontariens ont, selon Dorais, toutes les chances de produire le renouveau dont notre culture abâtardie a un urgent besoin. Pourquoi l'Ontario français? Parce que le Franco-ontarien est au bout de son rouleau, parce qu'il est, symboliquement et politiquement, le "dernier des Hommes," contrairement au Québécois, son frère assis entre deux chaises, incapable dassumer la dépossession totale qui est sa vérité occultée et qui, si elle était enfin admise, le conduirait à la fête de son indépendance par rapport à toutes les forces de l'oppression.

Or cette fête primitive, désarmante dans sa splendide simplicité, Dorais en voit en 1968 les signes en Ontario français, dans la jeunesse franco-ontarienne et dans sa contre-culture. Nombreuses sont à ce titre les descriptions de l'épisode libérateur des années soixante-dix à Sudbury, auquel Dorais a participé de plein front: 
Sudbury, ville minière, ville universitaire, ville marginalisée dans le grand Tout ontarien, ville d'exil pour celui qui venait (inexplicablement?) de quitter son Québec natal. Dans cette ville naissent avec une spontanéité apparente de nombreux instruments d'affirmation collective: un theâtre, une chanson, une maison d'édition, une manifestation politique dont l'emphase porte sur la participation de tous. Dorais décèle dans cette émergence plus qu'un simple événement historique, plutôt une véritable métamorphose collective, les premiers signes de la libération tant attendue (après tout, le premier recueil des éditions Prise de Parole ne s'intitulait-il pas Lignes-signes en 1973?), libération inscrite chez Dorais dans le mouvement théorisé de tous les peuples opprimés à travers le monde vers le renversement des forces de domination.

Toute l'Histoire - et Dorais entend surtout les échos de l'histoire européenne - semblait ici se résumer et s'éblouir. Ce sont ces curieux raccourcis historiques, dont les textes de Dorais sont remplis, où, par la magie de l'écrivain, l'héritage philosophique de Kant à Nietzsche, la pensée intellectuelle de Bossuet à Voltaire, les grandes idéologies françaises de notre siècle ne participent plus à notre humiliation, mais annoncent plutôt - et Dorais s'émerveille!la Fête dont nous serons, marginalisés, les centres reconquis.

Et il y a plus. Il y a des conditions historiques qui définissent l'Ontario français et le distinguent des autres cultures minorisées. C'est que les Franco-ontariens n'ont pas de territoire à délinéer et à défendre. Ils ne sont pas entravés par lui, comme le sont peut-être les Québécois. Les Franco-ontariens sont partout et nulle part; c'est pourquoi leur lieu d'épanouissement ne sera donc de toute évidence qu'un discours. Rien de plus et rien de moins. En 1982, Fernand Dorais rêve encore: "Rassembler notre géographie est-ce encore possible? Nous échouons là où des Asiatiques, des Italiens, des Israélites réussissent. Compenser l'espace physique par l'espace culturel semble pour l'heure la voie à emprunter" (EMS, p.68). Faute de pouvoir se regrouper géographiquement, dans cet espace sudburois qui dans le rêve d'un instant pourrait être le nôtre - mais comment imaginer que l'élite outaouaise ou torontoise accepte d'émigrer vers le Nord? - il faudra que l'Ontario français puisse se développer avant tout un espace de culture. En fait, n'est-ce pas là la plus grande découverte et la plus grande illusion de la nouvelle 
école sudburoise en 1968? N'est-ce pas hors des contraintes de l'espace que se produit pour les marginalisés absolus la Fête collective de la libération? Dorais le croit en tout cas fermement. "Le tissu franco-ontarien a toujours tiré sa plus grande force et sa moindre usure de sa texture socio-culturelle - sans cesse notre francophonie est renée de sa représentativité théâtrale et musicale, de ses 'séances' paroissiales et scolaires. La fête nous enfanta. Le spectacle de nous voir et dire nous ressource, reconnaissance d'une fraternité blessée. L'image et le son nous demeurent matrice communautaire" (EMS, p.75). Or, ailleurs, Dorais explique que l'essence de la dépossession dont souffre le Franco-ontarien, réside dans son exclusion du domaine du représentè. Le Franco-ontarien, comme tous les minorisés, ne se voit pas. La "fête" sudburoise était donc avant tout, aux yeux de Dorais, l'espoir d'un accès définitif à la représentation collective - définitif parce que nous en avons en nous les données matricielles - en dépit du déni de l'espace géographique qui fait notre condition d'existence.

Dorais décrit abondamment les différents moments de lutopie sudburoise dans un article lyrique, datant de 1989, publié pour la première fois dans Témoins d'errances. Des textes antérieurs avaient évoqué cette utopie dans un cadre plus théorique, mais en 1989 Dorais se laisse aller à la "réappropriation d'un pays-temps familier," (TE, p. 71). S'y trouvent rappelées les étapes de la création de CANO et ultérieurement du Théâtre du Nouvel-Ontario. Dorais s'y présente comme l'observateur privilégié et éminemment sympathique de CANO surtout, utopie communale de la culture marginalisée enfin sur la voie de l'auto-représentation. "CANO ... une commune au strict sens contre-culturel, un partage en amitié, une communion en situation de présence réciproque, qui rendit, et seule pouvait rendre possibles, la ou les créations qui suivraient" (TE, p.76). Ce moment sacral, exprimé en termes religieux, aura été bref, suivi d'un repli tactique vers la préservation et l'individualisme, "temps terne et gris où semblait aller s'enliser petit à petit dans les marais de l'indifférence généralisée" ( $T E$, p.80), mais il est pour Dorais fondateur de nos modes de représentation collective en Ontario français. Cet épisode sudburois, dont le grand prêtre sacrifié dans la personne d'André Paiement est la caution sacrale, devient notre origine, jusque là manquante, surtout le lieu d'origine du littèraire dans notre socièté 
si privée d'images d'elle-même. "Ainsi, naquimes-nous ... Ainsi, en trois temps, a éclos notre modernitẻ problématique! J'en atteste" (TE, p.81). Ainsi a parlé le chroniqueur, à près de vingt ans de distance, au moment où il ne reste guère pour lui de cette modernité qu'un narrateur par procuration qui n'a plus le courage (le droit) de parler à la première personne.

Nous en arrivons presque au littéraire. Car avec Dorais nous savons que le littéraire est notre destination finale. Mais avant, et pour justement s'en approcher, il faut retourner un instant encore à février 1978, au texte premier d'Entre Montréal et Sudbury, consacré à André Paiement, fondateur et animateur de CANO, qui, après sa mort prématurée, ètait devenu pour tous une véritable légende. Fernand Dorais ne conteste nullement cette légende; au contraire, il la conforte; il confirme le statut héroïque d'André Paiement et fait de lui l'image en raccourci de notre double participation au monde de la dépossession et à celui de la fête. Ce qui frappe dans ce texte liminaire, ce n'est pas tant son titre - "Qui a tué André Paiement?" - car il est sûr que son auteur a beaucoup, très profondément, aimé Andrè Paiement; c'est plutôt l'absence de la figure de Paiement, hormis le titre et les deux paragraphes finals, dans un article qui devrait en être le panégyrique. C'est que Paiement reste très purement le pré-texte; il n'est pas le texte lui-mème qui s'annonce toujours tragiquement à faire. ${ }^{9}$

Or ce document 1 donne à Fernand Dorais l'occasion de développer à nouveau son anthropologie de la minorisation. Car la mort si tragique d'André Paiement, c'est tout cela qui définit la condition d'existence du minoritaire: l'aliénation économique, les fidélités conflictuelles, la double appartenance au langage, l'indécision et la honte de l'origine. Dorais ne peut que faire de Paiement une métaphore de l'existence collective et une confirmation de notre libération éventuelle par la représentation discursive. Paiement est mort: vive le théâtre! Car il fallait que toute cette panoplie de nos misères soit représentée, au risque de sombrer par le fait même dans la folie et dans la mort. C'était le risque à prendre. Il n'y avait pas de choix, dira Dorais. C'est ce qui fait la grandeur de Paiement, que cette mort par le suicide (collectif?) puisse engendrer la théorie: "on a du pouvoir ou on n'y a pas accès; et les acculturés ici sont maintenus à l'êcart. Ils doivent donc y et en participer, sous 
risque fatal de demeurer les aliénés du pouvoir en leurs propres réalités socio-économiques. Y participant, ils consentent (...) à jouer le jeu et selon les règles de l'occupant, qui, dorénavant, de l'avantscène et mieux de la coulisse, impose son langage. Langage et jeu du pouvoir dont l'acculturé parvient difficilement à comprendre les subtilités et les ficelles. La logique du langage dominant lui èchappe, aussi joue-t-il toujours perdant" (EMS, p.20). Ainsi, c'est ce sentiment d'échec collectif qui resplendit dans l'acte final du théâtre d'André Paiement. "La compulsion d'échec est le lot de l'acculturé: s'il en coûte de n'être pas vainqueur, on paie encore plus cher d'être vaincu. Son châtiment c'est 'soi-même'" (EMS, p.23).

La fête sudburoise a finalement connu son supplicié. Rien ne sert de chercher le coupable ailleurs. Cette mutilation du collectif était inscrite depuis toujours dans l'histoire du minorisé. L'Ontario français, en quête de culture, ne pouvait y échapper. Ces textes cruciaux de Dorais, avec tout ce qu'ils comportent de mystification, établissent la place génésique de l'expérience culturelle sudburoise dans le discours franco-ontarien actuel. S'agit-il d'une illusion? Sans aucun doute, mais il en reste que cela n'a aucune importance. Nous sommes toujours dans le domaine du représentè.

Mais parlons encore de Sudbury. Au delà de ces considérations plus générales, c'est bien cet espace sudburois qui acquiert une représentation privilégiée (le privilège de la représentation?) chez Dorais. Reprenant une symbolique très connue en 1960 au Québec, Dorais fait du Nord le pôle de la revendication franco-ontarienne, le lieu de rassemblement d'un peuple dont la caractéristique principielle est la dispersion. Ce Nord mystifiant, que toute une panoplie d'auteurs franco-ontariens reprendront après Dorais, comme s'il était une pure évidence, résume à lui seul les caractéristiques psychologiques de l'isolement et de la marginalisation, et de leur renversement ultime - car le Nord rassemble - dans la fête. "Il faut tôt ou tard un Nord quelque part ..., ainsi qu'une clôture au sens. Il faut un 'lieu' où habiter, s'habiter, un 'milieu', pour devenir tout simplement "humain'." ${ }^{10}$ Encore ici, l'univers de Fernand Dorais est traversé par le déracinement, l'errance, le milieu (entre Montréal et Sudbury) qui n'en est pas un. D'ailleurs le texte que je viens de citer, peuplé de guillemets et d'hésitations, montre bien la particularité un peu étrange de l'espace culturel proposé ici. Le véritable milieu, 
auquel devrait aspirer l'Ontario français, ne se loge pas à mi-chemin entre le Québec et l'Ontario (il faut avoir le courage de s'avancer plus loin), mais en plein centre de sa géographie mentale. Le sens du mot "Nord" ne peut en être autrement.

A ce sujet, si Fernand Dorais reste pour nous un témoin essentiel du développement, à partir de 1970, d'une institution culturelle autonome en Ontario français, c'est qu'il est aussi celui qui a le mieux articulé les rapports èminemment ambigus que les Francoontariens entretiennent alors avec les Québécois. Dans un bref article de Témoins d'errances, intitulé "L'Ontario francophone avant et après 1967," Dorais tente de retracer l'histoire de ce qu'il appelle le schisme entre le Québec et l'Ontario français. Il existait d'abord une phase nostalgique. "Avant, on vivait à l'heure de Montréal" (TE, p.65), dans une continuité avec la production culturelle québécoise, dont l'Ontario français ne se considérait à vrai dire qu'une extension; "Un Québec qui s'émancipe, un humanisme inouï, inédit, qui semblait naître: voilà le contexte de la seconde naissance, ou si l'on veut présence, des francophones en Ontario" (TE, p.67). Mais l'histoire, pour Dorais, n'est qu'une recherche d'autonomie, une prise en mains des peuples par eux-mêmes, dans une longue procession d'indépendances partout dans notre univers géo-politique. Et le Québec, affamé d'autonomie, seule terre d'asile des francophones en Amérique du Nord, a fini par forcer sa diaspora à se redéfinir douloureusement: "Pour la première fois, un schisme s'opéra dans la 'parentè', entre les cousins. Ceux qui voulurent rester [en Ontario], l'immense majorité, durent alors songer à se trouver une identité culturelle spécifique, déliée quelque peu de celle bruyamment proclamée au Québec" (TE, p.68). Cette coupure du lien de parenté n'a pas l'effet catastrophique qu'on pourrait imaginer. Dorais y voit, comme d'autres chroniqueurs du mouvement culturel sudburois, le principe d'une autonomie institutionnelle de l'Ontario français, une autonomie dont les Franco-Ontariens n'auraient sans doute eu ni l'idée, ni le courage, n'eût été de l'indifférence brutale dans laquelle le Québec les a plongés après 1970 .

Conjoncture historique et interprétations littéraires forment donc, chez Dorais, un tout indissociable, un heureux mélange de fatalité et d'autodétermination. Il fallait bien que quelque chose arrive pour que démarre la nouvelle entreprise culturelle dont Dorais 
se fait le narrateur assidu. Il fallait une origine. Cette origine restituée, même vécue dans la blessure d'une mère-patrie qui semble abandonner sa progéniture à l'indifférence, suffit à lancer l'oeuvre d'art sur la voie de l'autonomie. Dorais insiste à maintes reprises sur la propension de l'oeuvre artistique à promouvoir les conditions de la liberté. C'est comme si cela advenait de soi. "Que partout et toujours l'oeuvre d'art opte pour le parti de la liberté et de la libération" (TE, p.138). Libération individuelle, de tous les sévices affectifs de la minorisation, et libération collective, de toutes les contraintes socio-politiques qui conduisent à l'humiliation. "Tel aura èté le cas en Ontario francophone, au début de sa production autonomisante, séparé enfin du Québec. Nous avons commencé ici par un cri, à tout point de vue assez sauvage il est vrai (et il le faut): le reste viendra par surcroît" (TE, p.139). Par surcroît, en effet, puisque, pour Dorais, le renversement des conditions minoritaires fait partie d'un mécanisme qui, une fois déclenché (ici par le retrait stratégique du Québec) ne peut plus être arrêté.

Il y a donc, à n'en pas douter, une revendication triomphante chez Dorais, qui paraît bien être l'envers de la longue errance si souvent décrite dans ses oeuvres, comme si en fin de compte c'était l'Ontario français, dans sa pure et jeune marginalité, qui finirait par surclasser le Québec. Dans un article inédit mentionné plus tôt, Dorais réserve pour le Québec ses jugements les plus acerbes: "... que foutent les Québécois, race maudite s'il en est une! A genoux, à genoux, songe ça, quêtant des restes de dignité qu'on va même jusqu'à lui refuser!" (p.4) Mais cette hargne contre un Québec jugé trop indécis ne dure pas. Le ça se laisse doucement encore une fois couler dans le nous indistinct. Eux et nous, qu'y a-t-il donc pour nous séparer? Dorais a beau tirer de tout cela des leçons d'autonomie triomphante; il en reste que la littérature franco-ontarienne ne peut pas ne pas voir dans le Québec la figure un peu plus colossale, il est vrai, un peu plus incongrue, de sa propre humiliation. Ces liens-là, Dorais ne les coupe jamais.

Dans toute cette oeuvre, il demeure une constante qui ne se dément jamais, celle de l'absolue présence du littéraire. En fait, la littérature, parce qu'elle est ni plus ni moins un discours, est le mode d'existence privilégié des peuples minoritaires. Comment cela se fait-il? Dorais l'explique dans le tout dernier texte de Témoins 
d'errances. Trois choses fondent le littéraire et sa relation avec l'existence minoritaire. D'abord, la littérature a tendance à s'institutionnaliser très rapidement. C'est ce qui s'est produit au Québec même, après 1950; le même phénomène se produit depuis vingt-cinq ans en Ontario français. Mais, plus qu'une production et une consommation de livres, une litterature engendre aussi pour Dorais une structure sociale qui permet la représentation collective: une critique universitaire (encore embryonnaire en Ontario), des centres de recherches, des maisons d'édition, des lieux de spectacles. Ensuite, cette institution doit se mettre immédiatement à l'oeuvre de la libération. Ce lien avec la liberté, très faible au fond car il n'est qu'un article de foi chez Dorais, justifie la présence centrale de la littérature dans toute argumentation des peuples humiliés vers leur libération. Enfin, la littérature n'est-elle pas toujours par essence une entreprise minoritaire? Mimant donc parfaitement le statut et les revendications des minorisés, ne s'insère-t-elle pas dans son humiliation quotidienne, non pas pour'y apitoyer, mais pour la faire éclater? "... toujours le créateur, pris dans et par son imaginaire, prend position pour le minoritaire en soi, sa dignité et sa fierté, proclamant l'autonomie absolue d'une liberté contre toute dépendance et tout ce qui s'essaie à bâillonner sa voix" (TE, p.137).

Ces très beaux textes des dernières pages de Témoins d'errances semblent ainsi permettre à Dorais l'élaboration d'une théorie littéraire de la minorisation, qui ne sera pas seulement vécue dans le détachement de l'anthropologie, la reléguant alors à l'état passif de folklore, mais comme production artistique vivante, originale et surtout essentielle au devenir de toute l'humanité. On aura beau voir dans ces pages une exagération, la force de leur conviction presque théologique ("instaurer tout à son image et à sa ressemblance dans la libertè," TE, p.139) fait verser l'oeuvre de Dorais dans le discours sacré. Nous le savions pourtant depuis le début, depuis les premières interventions. Car les descriptions de la minorisation, dans les articles de 1978, annonçaient déjà l'éventuel lien sacral avec la genèse du monde, "patrie antérieure de toute liberté, terrain d'élection de l'autonomie" (TE, p.148). C'est que le littéraire pour Dorais ne ment pas: il est d'une fidélité absolue à son origine. En fait, il est le discours toujours recommencé de l'origine. C'est en cela 
qu'il est si crucial pour les sans-patrie que sont les Franco-ontariens et tous les autres peuples minoritaires.

La conclusion nous renvoie au Document 4 d' Entre Montréal et Sudbury, l'un des plus denses et des plus profonds de toute l'oeuvre de Fernand Dorais. Dans cet article, tout en se référant à l'écriture française de l'après-guerre et à l'oeuvre entre autres d'Emile Nelligan, Dorais situe la minorisation dans les contours plus généraux d'une théorie du désir et de la violence. "Mais comment devient-on aberrant? Entre la Folie au Pouvoir, fonctionnelle et institutionnalisée, et la folie carcérale, institutionnalisée mais non opératoire, se tient la troisième folie, celle d'être aux coordonnées impossibles. Sa marque me paraît être l'Angoisse" (EMS, p.63). Si l'avenir appartient aux Humiliés, c'est qu'ils sont les seuls à maîtriser parfaitement les conditions de la troisième voie de la folie, celle de l'angoisse et de la peur. Ce qui rend pour Dorais la littėrature franco-ontarienne si passionnante au sortir des années soixante-dix, c'est sa manière de théâtraliser la peur collective et de l'institutionnaliser, pour enfin la débloquer et la libérer. "Le Marginalisé est aussi, en dehors de toute foi, la chance de l'Univers, la plaie dans la parturition de ses métamorphoses obligées" (EMS, p.78). Tout est là de ce qui sera pour Dorais la Fête de ceux qui sont maîtres de la métamorphose. Le philosophe n'y va pas de mains mortes. C'est son droit absolu. La patrie du Franco-ontarien, c'est son discours.

\section{Notes}

${ }^{1}$ Raymond Quatorze, La Prison rose bonbon (Sudbury: Prise de Parole, 1990). Qui donc est Raymond Guatorze?

${ }^{2}$ Ibid., p.11

${ }^{3}$ Ibid., p.88

${ }^{4}$ Fernand Dorais a publiê deux collections d'articles et de communications. Les deux titres évoquent l'errance, la dérive: Entre 
Montréal et Sudbury, prétextes pour une francophonie ontarienne (Sudbury: Prise de Parole, 1984) et Témoins d'errances en Ontario français (Hearst: Le Nordir, 1990). Les deux recueils sont notés EMS et $T E$ respectivement dans le présent article.

${ }^{5}$ Entre Montréal et Sudbury, p.49, n. 3.

${ }^{6}$ Cet article encore inédit, intitulé "Ça-Moi-l'Autre-... et les Autres en situation de multiculturalisme," a été préparé pour la revue Francophonies d'Amérique. Mais, dans une lettre personnelle du 21 août 1991, Dorais exprimait des doutes sur la publication éventuelle de ce texte.

${ }^{7}$ Franz Fanon. Les Damnés de la terre (Paris: Maspéro, 1961) et Albert Memmi. Portrait du colonisé, dont Dorais consulte l'édition québécoise (Montréal: L'Étincelle, 1972).

${ }^{8} C f$. aussi l'article intitulé "Minorité, autonomie, dépendance," dans Témoins d'errances en Ontario français, où Dorais décrit ainsi les conditions de la minorisation: "Etre prudent surtout, ne pas provoquer inutilement, ne susciter d'antagonisme aucun et dissimuler, refouler les différences. On peut toujours finir et on finit toujours par s'entendre" (p.141). Ce type de discours ironique abonde dans toute l'oeuvre de Dorais.

${ }^{9}$ Il est intéressant que Robert Dickson, dans Abris nocturnes (Prise de Parole, 1986), ait cru bon de recourir au stratagème d'un cahier fictif, devant avoir appartenu à André Paiement, que le poète s'apprêté maintenant à parachever. André Paiement apparaît ici clairement comme le pré-texte d'Abris nocturnes.

${ }^{10}$ "Ça-Moi-l'Autre," p.4. 